\title{
Coronary Artery Bypass Grafting in Patients with Systemic Lupus Erythematosus
}

\author{
Shi-Min Yuan \\ Department of Cardiothoracic Surgery, The First Hospital of Putian, Teaching Hospital, Fujian Medical University, Putian, Fujian \\ Province, China
}

\begin{abstract}
Surgical treatment of coronary artery disease in the systemic lupus erythematosus (SLE) patients has not been comprehensively addressed. The present review aimed to give an overview of coronary artery disease in the SLE patients receiving coronary artery bypass grafting (CABG). The study materials were based on comprehensive literature retrieval, which recruited 17 pertinent articles with 30 patients. No differences were found in the graft patencies between the arterial and venous grafts; and between the early and late patency rates. Pathological inspections revealed that all graft vessels were normal with no signs of SLE-related atherosclerosis or vasculitis, one coronary artery was pathologically normal, and another coronary artery showed vasculitis. The coexisting disorders, including diabetes mellitus, hyperlipidemia, and nephropathy in the SLE patients cause early deterioration of the saphenous vein grafts. Early occlusion of the saphenous vein grafts was also observed in SLE patients. The left anterior descending coronary artery was most commonly affected by SLE and was the most common coronary artery requiring a CABG procedure. The graft vessels, both arterial and venous, rarely degenerated; whereas, early and late graft failure was usually caused by technical failures. The lack of vasculitis and atherosclerosis in the arterial grafts encourage surgeons to prefer to use the arterial grafts in SLE patients. Less invasive surgical technique would favour the patients in terms of long-term outcomes.
\end{abstract}

Key Words: Coronary artery bypass grafting, Graft occlusion, Vascular, Systemic lupus erythematosus.

How to cite this article: Yuan SM. Coronary Artery Bypass Grafting in Patients with Systemic Lupus Erythematosus. J Coll Physicians Surg Pak 2020; 30(09):961-965.

\section{INTRODUCTION}

Cardiovascular involvements of systemic lupus erythematosus (SLE) may include pericarditis, myocarditis, endocarditis, valvular disorders, and coronary artery disease. ${ }^{1}$ Coronary artery disease is a leading cause of death of SLE patients. ${ }^{2}$ It can be attributed to premature atherosclerosis, arteritis, thrombosis, embolisation, vasospasm, and impaired coronary flow reserve. ${ }^{3}$ Myocardial infarction can be a presenting manifestation of SLE. ${ }^{4}$

It was reported that angina/myocardial infarction occurred in $2-16 \%$ of the patients. ${ }^{3}$ A necropsy observation on 623 coronary segments from 22 necropsy patients showed that the prevalence of coronary artery stenosis of different degrees was significantly higher than that of the control. ${ }^{5}$ In addition, the development of coronary artery aneurysm in SLE patients can also be a sequel of arteritis and premature atherosclerosis. ${ }^{4}$

Correspondence to: Shi-Min Yuan, Department of Cardiothoracic Surgery, The First Hospital of Putian, Teaching Hospital, Fujian Medical University, Putian, Fujian Province, China

E-mail: shiminyuan@126.com

Received: May 15, 2019; Revised: November 01, 2019;

Accepted: December 04, 2019

DOI: https://doi.org/10.29271/jcpsp.2020.09.961
Nevertheless, surgical treatment of coronary artery disease in SLE patients is sporadically reported and the results of coronary artery bypass grafting (CABG) in these patients are somewhat inconclusive. The present study aimed to review the available evidence on coronary artery disease in SLE patients receiving a surgical treatment.

\section{METHODOLOGY}

The preferred reporting items for systematic reviews and meta-analyses (PRISMA) statement guidelines were followed in this meta-analysis. Publications were systematically searched in the PubMed, Highwire Press, and the Cochrane Library databases. The MeSH terms and keywords used to identify articles included systemic lupus erythematosus, coronary artery disease, myocardial infarction, coronary artery bypass grafting, off-pump coronary artery bypass, and minimally invasive direct coronary artery bypass (MIDCAB). Bibliographic references were also tracked for the completeness of the literature retrieval. Thirty-six articles were found related to the topic and keywords in the literature search; and 17 articles, which met the inclusion criteria during preliminary assessment, were included in the review. Of them, articles with a patient population receiving $C A B G$ procedure in the majority (for example, $>90 \%$ ) of patients and receiving CABG and concurrent valve procedures in a minonity of patients were recruited into this 
study. The exclusion criteria were articles describing coronary artery disease in rheumatic patients $(n=5)$, cardiovascular risks of SLE patients $(n=4)$, irrelavant design $(n=2)$, gene polymorphism of SLE patients $(n=2)$, ischemic stroke of SLE pateints $(n=1)$, hospitalisation of SLE patients receiving CABG $(n=1)$, lupus nephritis $(n=1)$, background discussion of SLE $(n=1)$, cardiovascular diseases in connective tissue disorder $(n=1)$, and cardiovascular diseases in Sjögren syndrome patients $(n=1)$.

Data were carefully extracted for details of the study population, demographics, medical history of SLE, clinical manifestations, duration of steroid use, surgical techniques, bypass graft configurations, early ( $2-3$ weeks after operation) and late ( $\geq 1$ year) graft patency (detected by coronary angiography), pathology of the graft vessels and coronary arteries, length of follow-up, and patients' prognoses.

Quantitative data were presented as mean \pm standard deviation, and the intergroup differences were compared by independent samplest-test. The categorical variables, expressed by numbers and percentages, were compared by Fisher's exact test. An IBM SPSS statistics software version 22.0 was used for the statistical analyses. $p<0.05$ was considered statistically significant.

\section{RESULTS}

In total, 17 articles were obtained with 30 patients involved in the articles. ${ }^{4,6-20}$ Gender was not indicated for one of the patients. Of the remaining 29 patients, 10 (34.5\%) were male and 19 (65.5\%) were female patients $\left(\chi^{2}=5.6, p=0.035\right)$, with a male-to-female ratio of $1: 1.9$. The patients were at the age of $50.6 \pm 16.3$ years $(n=28)$. There was no difference in patients' age between the male and the female patients $(60.0 \pm 13.0$ years vs. $51.9 \pm 13.1$ years, $p=0.167)$. The SLE history of the patients lasted $11.5 \pm 7.4$ years $(n=20)$. The female patients had a longer SLE history than the male patients ( $15.4 \pm 6.9$ years vs. $7.4 \pm 5.1$ years, $p=0.038$ ). The steroid use was $12.4 \pm 4.5$ years $(n=8)$ for the patients until current admissions.

Clinical manifestations for current admissions were described in $13(43.3 \%)$ patients. There were 19 manifestations in the 13 patients, including chest pain/angina $(n=12,63.2 \%)$, ${ }^{4,6,8,9,11-15,17}$ dyspnoea/shortness of breath $(n=5,26.3 \%),{ }^{8,11,12,15,16}$ epigastric pain $(n=1,5.3 \%),{ }^{8}$ and numbness $(n=1,5.3 \%){ }^{8}$ One of the patients with coronary artery aneurysm of the right coronary artery developed myocardial re-infarctions. ${ }^{4}$

Surgical indication for CABG was coronary artery stenosis or occlusion in 29 (96.7\%) patients; and coronary artery aneurysm associated with coronary artery stenosis in one (3.3\%) patient. Two (6.7\%) patients were operated on an urgent basis. ${ }^{8,11}$ The number of the diseased coronary arteries was described in 23 (76.7\%) patients, in whom 2-vessel disease predominated (Figure 1). The affected coronary artery was described in 17 (56.7\%) patients, with the left anterior descending coronary artery being the most commonly affected coronary artery (Figure 2). The number of the bypass grafts was $2.3 \pm 1.1$ per patient $(n=24)$.

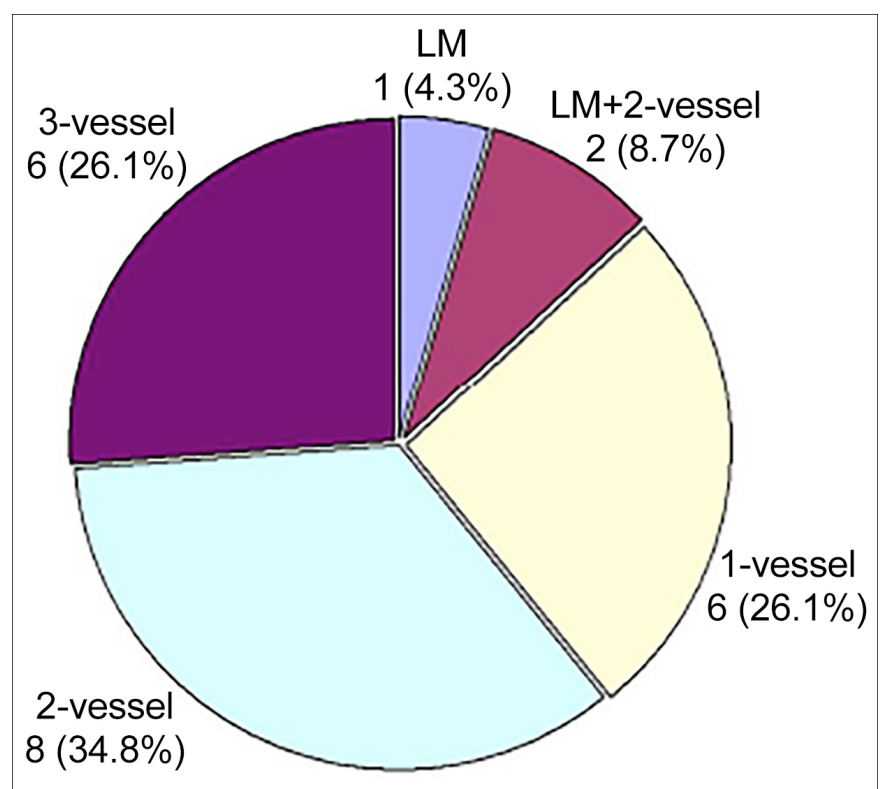

Figure 1: The distribution of the number of the diseased coronary arteries in 23 patients. LM: Left main trunk.

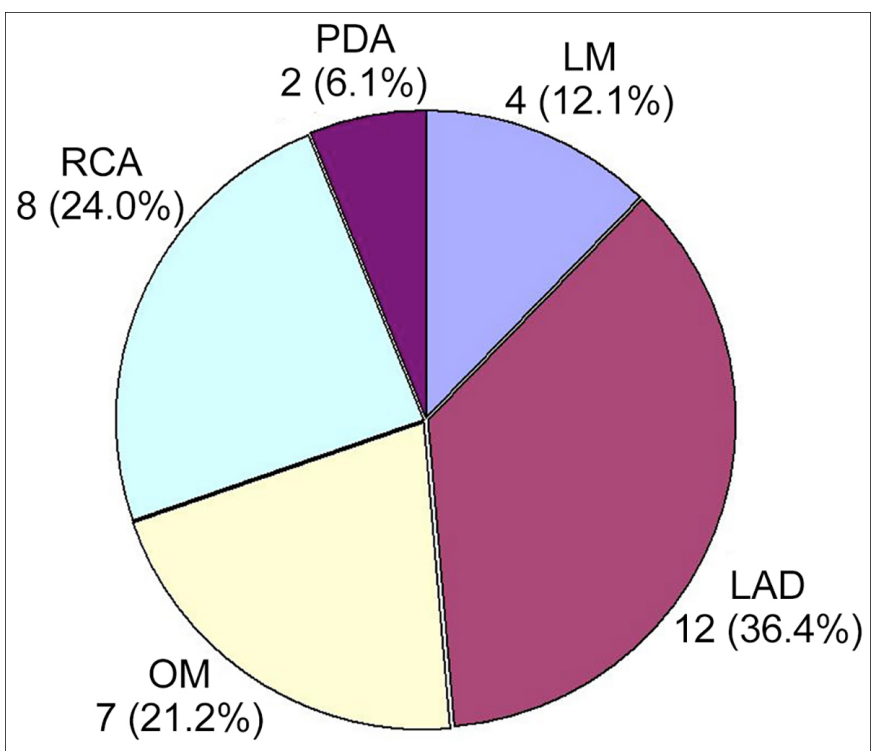

FIgure 2: The affected coronary artery in 17 patients. LAD: left anterior descending coronary artery. LIMA: Left internal mammary artery; OM: Obtuse marginal branch; PDA: Posterior descending coronary artery; RCA: Right coronary artery.

The bypass technique was not datailed in one report for one patient. In the remaining 29 patients, the techniques were conventional CABG in 22 (75.9\%) patients, ${ }^{4,6-10,13,15-19}$ on-pump beating heart CABG in $3(10.3 \%)$ patients, ${ }^{10}$ off-pump coronary artery bypass in 3 (10.3\%) patients, ${ }^{6,11,14}$ and endoscopic coronary artery bypass in 1 (3.4\%) patient. ${ }^{12}$ The coronary artery bypass grafts were substantially described in 20 (66.7\%) patients, in whom 45 grafts were bypassed, with 40 (88.9\%) single grafts, $3(6.7 \%)$ sequential grafts, ${ }^{16,18}$ and $1(2.2 \%) \mathrm{Y}$ graft. ${ }^{14}$ The left internal mammary artery-left descending coronary artery bypass was the most common bypass configuration of this patient setting $(28.9 \%, 13 / 45)^{6,11-19}$ 
Table I: Distributions of $\mathbf{4 7}$ graft (donor) vessels and 51 receipt vessels for coronary artery bypass grafting.

\begin{tabular}{|c|c|}
\hline Vessel & n (\%) \\
\hline \multicolumn{2}{|l|}{ Graft (donor) vessel } \\
\hline Left internal mammary artery ${ }^{6,7,11-19}$ & $16(34.0)$ \\
\hline Right internal mammary artery ${ }^{14,18}$ & $4(8.5)$ \\
\hline Right gastroepiploic artery ${ }^{13}$ & $1(2.1)$ \\
\hline Saphenous vein graft $^{4,6,7,11,15-18}$ & $26(55.3)$ \\
\hline \multicolumn{2}{|l|}{ Receipt vessel } \\
\hline Left anterior descending coronary artery $y^{6,8,11-19}$ & $19(37.3)$ \\
\hline Diagonal branch ${ }^{14-16,18}$ & $7(13.7)$ \\
\hline Circumflex branch ${ }^{8,18}$ & $7(13.7)$ \\
\hline Obtuse marginal branch ${ }^{6,14-17}$ & $7(13.7)$ \\
\hline Left main trunk $^{8}$ & $1(2.0)$ \\
\hline Right coronary artery ${ }^{4,11,13,18}$ & $7(13.7)$ \\
\hline Posterior descending coronary artery ${ }^{15,17}$ & $3(5.9)$ \\
\hline
\end{tabular}

Table II: Acomparison of graft patency.

\begin{tabular}{|l|l|l|l|l|}
\hline Graft patency & Early & Late & $\boldsymbol{\chi}^{2}$ & p-value \\
\hline Arterial & $9 / 11(81.8)$ & $7 / 8(87.5)$ & 0.1 & 1.000 \\
\hline Venous & $16 / 18(88.9)$ & $6 / 6(100)$ & 0.7 & 1.000 \\
\hline Unspecified & $4 / 7(57.1)$ & $1 / 1(100)$ & 0.7 & 1.000 \\
\hline Overall & $29 / 36(80.6)$ & $14 / 15(93.3)$ & 1.3 & 0.409 \\
\hline $\begin{array}{l}\chi^{2} \text { (arterial vs. venous } \\
\text { graft) }\end{array}$ & 0.3 & 0.8 & -- & -- \\
\hline $\begin{array}{l}\text { p-value (arterial vs. } \\
\text { venous graft) }\end{array}$ & 0.622 & 1.000 & -- & -- \\
\hline
\end{tabular}

There were 47 graft vessels reported, including 21 (44.7\%) arterial and $26(55.3 \%)$ venous grafts $\left(\chi^{2}=1.1, p=0.409\right)$. The left anterior descending coronary artery was the most common coronary artery to be bypassed (Tablel).

Nine (30\%) patients had one or more concurrent/subsequent/late cardiac operations, including concurrent mitral valve replacement, aortic valve replacement, or mitral/tricuspid valvuloplasty $(n=5),{ }^{10,18,19}$ a pical aneurysmectomy $(n=1),{ }^{9}$ and stent deployment (to seal the coronary artery aneurysm and to dilate the stenotic lesion) $(n=1),{ }^{4}$ subsequent stent to the first diagonal branch on postoperative day $3(n=1),{ }^{12}$ and late aortic valve replacement three years after the initial CABG $(n=1) .{ }^{16}$

The graft patency was evaluated early in 19 patients and late in six patients. No differences were found in the graft patencies between the arterial and venous grafts or between the early and late patency rates (Table II). Twenty risk factors leading to graft failure were described in 16 (53.3\%) patients, with hypertension ${ }^{10,12,17,18}$ being the most common risk factor accounting for $35 \%$ (7/20), followed by renal failure $(20 \%, 4 / 20),{ }^{6,18}$ diabetes mellitus $(15 \%, 3 / 20),{ }^{17,18}$ antiphospholipid syndrome (10\%, $2 / 20),{ }^{14,19}$ hypercholesterolemia $(10 \%, 2 / 20),{ }^{4,18}$ positive lupus anticoagulant $(5 \%, 1 / 20),{ }^{19}$ and pulmonary embolism $(5 \%$, 1/20). ${ }^{14}$ The coexisting disorders, including diabetes mellitus, hyperlipidemia, and nephropathy, led to an early deterioration of the saphenous veingrafts. ${ }^{18}$

There were eight graft vessels (5 left internal mammary arteries, ${ }^{6,13,15}$ one right gastroepiploic artery ${ }^{13}$ and two saphenous vein grafts ${ }^{6}$ ) and two coronary arteries under pathological inspections in six (20\%) patients. All eight graft vessels were normal with no signs of SLE-related atherosclerosis or vasculitis, whereas one coronary artery was pathologically normal, ${ }^{15}$ and another coronary artery showed changes of vasculitis. $^{12}$

Patients were under a follow-up of $34.4 \pm 31.6$ months $(n=18)$. The prognoses were described in 29 patients: 17 (58.6\%) patients recovered, ${ }^{6,10,12-19} 2$ (6.9\%) patients were improved, ${ }^{8,9} 5$ (17.2\%) patients were complicated (early graft thrombosis, ${ }^{10}$ bleeding, ${ }^{4}$ mediastinitis, ${ }^{11}$ and superficial wound abscess of the right leg with subsequent saphenous vein graft occlusion ${ }^{15}$ ), and 5 (17.2\%) patients died..$^{10,18,19}$ There were 2 early deaths ${ }^{18,19}$ and 3 late deaths, ${ }^{10,18,19}$ with an early mortality of $6.9 \%(2 / 29)$, and a late mortality of $11.1 \%(3 / 27)$.

\section{DISCUSSION}

The premature coronary atherosclerosis in SLE can be due to immune complex deposition and complement activation in the arterial wall, chronic steroid therapy that may worsen hyperglycemia and hyperlipidemia, and/or the associated antiphospholipid syndrome, which predisposes to thrombus formation and myocardial infarctions. ${ }^{12}$ The risk factors responsible for coronary artery disease in SLE patients include coronary arteritis and steroid-induced accelerated atherosclerosis. ${ }^{18}$ The SLE patients are usually young, and the acute coronary syndrome in these patients is largely attributed to the underlying autoimmune disease. ${ }^{12}$ The pathological changes of the coronary arteries in SLE patients can be atherosclerosis or arteritis, and the incidence of coronary atherosclerosis and arteritis wassimilar. ${ }^{21}$ Hyaline arteriolar disease seen in the coronary lesions probably causes myocardial infarction. ${ }^{21}$ Clinically, coronary arteritis, premature atherosclerosis, and SLE-induced myocardial microvasculopathy are considered to be the predisposing risk factors of myocardial infarction in SLE patients. ${ }^{14}$ Postoperative myocardial infarction can be due to graft thrombosis that may cause patient death. ${ }^{19}$

Balloon angioplasty procedures may pose some risks to these patients, as itmay dislodge the thrombus, resulting in an embolisation of the distal right coronary artery. ${ }^{4}$ This event may warrant an aggressive surgical operation in SLE patients.

CABG in SLE patients was first reported by Homcy et al. in $1982 .{ }^{9}$ In 2014, Maeda et al. described an SLE patient receiving an urgent CABG before the start of steroid therapy was associated with difficult perioperative management. ${ }^{11}$ Therefore, perioperative administration with methylpredonisolone and heparin was suggested for preventing from thrombus formation and aggravation of SLE. ${ }^{6}$ Moreover, in order to obtain a better operative course, the recommended perioperative management strategies included one-week preoperative suspension of anticoagulants to avoid operative bleeding; continuous administration of heparin to prevent from thrombus formation; activated clotting time controlled for $>500$ seconds during the operation and methylpredonisolone infusion to avoid acute aggravation of SLE; and early postoperative anticoagulant recovery. ${ }^{19}$

The SLE patients are at greater risks of postoperative morbidity 
and mortality. It was reported that the in-hospital mortality rate of CABG in SLE patients was 5.7\%. ${ }^{22}$ This result was much higher than in-hospital mortality rate of CABG in general population reported from The Society of Thoracic Surgeons Adult Cardiac Surgery Database, which was 1.7\% and 1.8\% in 2016 and in 2019 , respectively. ${ }^{23,24}$ Papa et al. identified four risk factors that predicted an increased morbidity of SLE patients, which were preoperative higher doses of steroid, multiple organ involvements, emergent operation, and renal failure. ${ }^{25}$ The SLE patients receiving CABG and concurrent valvular operation posed an increased risk of death. ${ }^{21}$ Uea et al. reported a hospital death of one of their SLE patients receving CABG was due to an urgent operation, acute heart failure, and a low ejection fraction of $26 \%{ }^{18}$

The patency rate of the arterial grafts in SLE patients receiving CABG was $81.8 \%$ (9/11) with two stenosed or string-like grafts due to intraoperative technical failures. There has been consensus on whether the arterial grafts should be preferred in SLE patients. ${ }^{26}$ In general population, the patency of coronary artery bypass grafts depends on graft choices, bypass configuration, and stenotic degree and location of the target coronary arteries. In the SLE patients, both coronary arteritis and atherosclerosis have been described in young SLE patients. The SLE patients were reported to often have vasculitis; and the incidence was reported to be $11-36 \% .{ }^{27}$ However, pathological inspections of the mammary arterial grafts revealed only minor atherosclerotic changes in most of the SLE patients, but no arteritis or significant atherosclerosis was noted. ${ }^{18}$ Ura et al. repeated angiography in two patients with the mammary artery grafting, which showed no signs of newly developed atherosclerosis or arteritis. ${ }^{18}$ The long-term patency of mammary artery grafts was demonstrated in various studies on SLE patients, but it was uncertain whether the mammary arterial grafts would develop arteritis and atherosclerosis in SLE patients at a later stage. Long-term patency of the gastroepiploic artery graft in patients with SLE also requires further evaluations. ${ }^{13}$ Moreover, the coexisting disorders, such as diabetes mellitus, hyperlipidemia, and nephropathy, often cause an early deterioration of the saphenous vein grafts in SLE patients. ${ }^{18}$ Early occlusion of the saphenous vein grafts has also been observed in SLE patients. ${ }^{28}$ The resistance of internal mammary artery to atherosclerosis was once attributed to prolonged steroid use in SLE patients, ${ }^{18}$ but nowadays it is convinced that the advantages of the internal mammary artery grafts over other conduits are due to the superior endothelial function, greater antithrombotic molecules, higher endothelial nitric oxide production, and flow remodeling. ${ }^{2,29,30}$ Most importantly, steroids, particularly long-term steroid use, inhibits the normal healing process and may predispose to aneurysmal formation in coronary artery disease patients. ${ }^{9}$ This may possibly interpret the graft failure in SLE patients.

The present study revealed that both young and old patients took shorter time to develop coronary artery disease than middle-aged patients. There was no discrepancy in the patency rates between the arterial and venous grafts. It proved that the graft vessels, both arterial and venous, rarely degenerated, and technical failures might be a major determinant of early and late graft failure. ${ }^{18}$ Meanwhile, the pathological changes of the coronary artery were noted in part of SLE patients.

Patients with SLE is often associated with an antiphospholipid syndrome, a severe prothrombotic disorder that may occur after surgical operations. The SLE patients receiving a heart operation by using a minimally invasive approach may prevent severe tendency of haemorrhage in relation to anticoagulation from happening. ${ }^{31}$ With hybrid CABG and coronary angioplasty, the outcomes of the SLE patients with coronary artery disease seem to be promising. ${ }^{18}$

The limited information from the pertinent literature constitutes the major drawback of this study. Comparisons of the surgical outcomes between the conventional and less invasive CABG procedures, and the graft patency rates between different grafts could not be sufficiently evaluated. Further observations of SLE patients receiving CABG are warranted for obtaining more conclusive results in terms of the graft patency, the pathological changes of the grafts and the coronary arteries, and the predisposing risk factors for graft failure of SLE patients.

\section{CONCLUSION}

SLE patients requiring CABG are mostly ladies. The left anterior descending coronary artery is the most commonly affected coronary artery that requires CABG. Prolonged steroid use in SLE patients enables the mammary arteries resistant to the atherosclerotic changes. The graft vessels, both arterial and venous, rarely degenerated; whereas, early and late graft failure was usually caused by technical failures. The lack of vasculitis and atherosclerosis in the arterial graft vessels encourage surgeons to prefer to use the arterial grafts in SLE patients. Less invasive surgical technique would favour these patients in terms of longterm outcomes.

\section{CONFLICT OF INTEREST:}

The author declared no conflict of interest.

\section{AUTHOR'S CONTRIBUTION:}

SMY: Substantial contribution to the conception and design of the work; acquisition, analysis, and interpretation of data for the work; drafting the work and revising it critically for important intellectual content; final approval of the version to be published; agreement to be accountable for all aspects of the work in ensuring that questions related to the accuracy or integrity of any part of the work are appropriately investigated and resolved.

\section{REFERENCES}

1. Miner JJ, Kim AH. Cardiac manifestations of systemic lupus erythematosus. Rheum Dis Clin North Am 2014; 40 (1):51-60.

2. Petri M. Detection of coronary artery disease and the role of traditional risk factors in the Hopkins Lupus Cohort. Lupus 2000; 9(3):170-5. 
3. Moder KG, Miller TD, Tazelaar HD. Cardiac involvement in systemic lupus erythematosus. Mayo Clin Proc 1999; 74(3):275-84.

4. Yoshikai M, Hamada M, Takarabe K. Coronary artery aneurysm with systemic lupus erythematosus. Jpn J Thorac Cardiovasc Surg 2004; 52:379-82.

5. Haider YS, Roberts WC. Coronary arterial disease in systemic lupus erythematosus: Quantification of degrees of narrowing in 22 necropsy patients ( 21 women) aged 16 to 37 years. Am J Med 1981; 70(4):775-81.

6. Bozbuğa N, Erentuğ V, Kaya E, Akinci E, Yakut C. Coronary artery bypass grafting in patients with systemic lupus erythematosus. J Card Surg 2004; 19(5):471-2.

7. De Matos Soeiro A, de Almeida Soeiro MC, de Oliveira MT Jr, Serrano CV Jr. Clinical characteristics and in-hospital outcome of patients with acute coronary syndromes and systemic lupus erythematosus. Rev Port Cardiol 2014; 33(11):685-90.

8. Galindo M, Chung L, Crockett SD, Chakravarty EF. Coronary artery disease in patients with systemic lupus erythematosus. Nat Clin Pract Rheumatol 2005; 1:55-9; quiz 60.

9. Homcy CJ, Liberthson RR, Fallon JT, Gross S, Miller LM. Ischemic heart disease in systemic lupus erythematosus in the young patient: report of six cases. Am J Cardiol 1982; 49(2):478-84.

10. Lin $\mathrm{CH}$, Lee $\mathrm{ML}$, Hsu RB. Cardiac surgery in patients with systemic lupus erythematosus. Interact Cardiovasc Thorac Surg 2005; 4(6):618-21.

11. Maeda K, Nishi H, Sakaguchi T, Miyagawa S, Ueno T, Kuratani $\mathrm{T}$, et al. Coronary artery bypass grafting in a patient initially presenting with systemic lupus erythematosus. Ann Thorac Cardiovasc Surg 2014; 20(5): 414-7.

12. Mehta PK, Samady H, Vassiliades TA, Book WM. Acute coronary syndrome as a first presentation of systemic lupus erythematosus in a teenager: Revascularization by hybrid coronary artery bypass graft surgery and percutaneous coronary intervention: Case report. Pediatr Cardiol 2008; 29(5):957-61.

13. Nishinaka $T$, Koyanagi $H$, Endo M, Nishida H. Coronary artery bypass in systemic lupus erythematosus using total autogenous arterial bypass. J Card Surg 1994; 9(4):440-2.

14. Park S, Hwang HY, Kang HJ, Kim KB. Myocardial revascularization in two patients associated with antiphospholipid syndrome: Different pathogenic patterns and angiographic results. Korean J Thorac Cardiovasc Surg $2011 ;$ 44(6):423-6.

15. Rinaldi RG, Carballido J, Betancourt B, Sartori M, Almodóvar EA. Coronary artery bypass grafting in patients with systemic lupus erythematosus. Report of 2 cases. Tex Heart Inst J 1995; 22(2):185-8.

16. Sakaguchi G, Minami K, Nakayama S, Tsuneyoshi H. Aortic valve replacement after previous coronary artery bypass grafting in a patient with antiphospholipid syndrome. Jpn J
Thorac Cardiovasc Surg 1998; 46(3):257-9.

17. Trivedi J, Padhy K, Kodem DR, Narasimham SBR, Satyanarayana PV. Coronary artery bypass grafting in patients with systemic lupus erythmatosus-a case report. Indian J Thorac Cardiovasc Surg 2009; 25:27-8.

18. Ura M, Sakata R, Nakayama Y, Ohtsuka Y, Saito T. Coronary artery bypass grafting in patients with systemic lupus erythematosus. Eur J Cardiothorac Surg 1999; 15:697-701.

19. Woś S, Bachowski R, Ceglarek W, Domaradzki W, Matuszewski M, Kucewicz E. Coronary artery bypass grafting without cardiopulmonary bypass-initial experience of 50 cases. Eur J Cardiothorac Surg 1998; 14 (Suppl 1):S38-42.

20. Yoshida M, Sasako Y, Kobayashi J, Minatoya K, Bando K, Kitamura S. Mitral valve plasty in systemic lupus erythematosus in the setting of antiphospholipid syndrome. Jpn J Thorac Cardiovasc Surg 2000; 48(6):391-3.

21. Doherty NE, Siegel RJ. Cardiovascular manifestations of systemic lupus erythematosus. Am Heart J 1985; 110(6): 1257-65.

22. Ward MM. Outcomes of hospitalizations for myocardial infarctions and cerebrovascular accidents in patients with systemic lupus erythematosus. Arthritis Rheum 2004; 50(10):3170-6.

23. D'Agostino RS, Jacobs JP, Badhwar V, Paone G, Rankin JS, Han JM, et al. The society of thoracic surgeons adult cardiac surgery database: 2016 update on outcomes and quality. Ann Thorac Surg 2016; 101(1):24-32.

24. D'Agostino RS, Jacobs JP, Badhwar V, Fernandez FG, Paone G, Wormuth DW, et al. The Society of Thoracic Surgeons Adult Cardiac Surgery Database: 2019 Update on Outcomes and Quality. Ann Thorac Surg 2019; 107(1):24-32.

25. Papa MZ, Shiloni E, Vetto JT, Kastner DL, McDonald HD. Surgical morbidity in patients with systemic lupus erythematosus. Am J Surg 1989; 157(3):295-8.

26. Bossert T, Falk V, Gummert JF, Rahmel A, Mohr FW. Coronary artery bypass grafting in patients with systemic lupus erythematosus. Z Kardiol 2003; 92:219-21.

27. Barile-Fabris L, Hernández-Cabrera MF, Barragan-Garfias JA. Vasculitis in systemic lupus erythematosus. Curr Rheumatol Rep 2014; 16(9):440.

28. Yamaguchi A, Kitamura N, Irie T, Otaki M, Minoji T, Tamura $H$, et al. A case of coronary artery bypass using saphenous vein for LMT lesion in a patient with systemic lupus erythematosus. Nihon Kyobu Geka Gakkai Zasshi 1988; 36(6):1008-11.

29. Yuan SM, Shinfeld A, Kachal E, Raanani E. The alternatives of autologous arterial grafts for coronary bypass surgery. Acta Cardiol Sin 2006; 22(3):121-33.

30. Otsuka F, Yahagi K, Sakakura K, Virmani R. Why is the mammary artery so special and what protects it from atherosclerosis? Ann Cardiothorac Surg 2013; 2(4):519-26.

31. Koster A, Ensminger S, Vlachojannis M, Birschmann I. Bivalirudin anticoagulation for minimal invasive transapical transcatheter aortic valve replacement in a patient with antiphospholipid antibodies. J Clin Anesth 2016; 33:373-5. 